\title{
Disseminated BCG-osis and the Importance of Performing Post-Mortem Examinations
}

\author{
Paul Andrew Lilburn ${ }^{1,2 *}$, Patrick Bazin², Alexander Beveridge², Emma Goeman ${ }^{3}$, Hazel Goldberg 1,3,4,5 \\ 'Prince of Wales Hospital, Barker Street, Randwick, Sydney, NSW, 2031, Australia \\ ${ }^{2}$ St Vincent's Hospital, 390 Victoria Street, Darlinghurst, Sydney, NSW, 2010, Australia \\ ${ }^{3}$ Royal Prince Alfred, Missenden Road, Camperdown, Sydney, NSW, 2050, Australia \\ ${ }^{4}$ Sydney Hospital, 8 Macquarie Street, Sydney, NSW, 2000, Australia \\ ${ }^{5}$ St George Hospital, Kogarah, NSW 2017, Australia
}

Article Info

\section{Article Notes}

Received: January 31,2019

Accepted: June 19, 2020

\section{*Correspondence:}

Paul Andrew Lilburn, Prince of Wales Hospital, Barker Street, Randwick, Sydney, NSW, 2031, Australia; Email: Paul.Lilburn@health.nsw.gov.au ; paul.lilburn2@svha.org.au

(c) 2020 Lilburn PA. This article is distributed under the terms of the Creative Commons Attribution 4.0 International License.

\section{Keywords}

Bacillus Calmette-Guerin (BCG) vaccine

BCG-osis

Mycobacterium bovis (M Bovis)

\section{Abstract}

Bacillus Calmette-Guerin (BCG) is a live-attenuated strain of Mycobacterium bovis ( $M$ bovis), originally developed as a vaccine against tuberculosis. It is also used in the treatment of bladder cancer. We present a case of disseminated BCG-osis which was only discovered at post-mortem. An 89-year-old male was admitted to a tertiary referral centre in inner Sydney in December 2015 with fevers, night sweats, exertional dyspnoea and $8 \mathrm{~kg}$ weight loss. The patient developed progressive type 1 respiratory failure requiring non-invasive positive pressure ventilation and treatment for heart failure, but he died eleven days into his admission. On microbiological testing Mycobacterium. tuberculosis complex (MTBC) DNA was detected in lung tissue, by polymerase chain reaction (PCR), using the Xpert ${ }^{\circ}$ MTB/RIF (Cepheid) platform, the day after the patient's death. Later mycobacterial culture and genomic deletion analysis identified the specific organism as $M$ bovis. This report describes an unusual presentation of systemic BCG-osis appearing more than three years after the last BCG instillation. This reinforces the fact that BCG instillation of the bladder for transitional cell carcinoma poses a potential risk of systemic spread even years after.

\section{Introduction}

Bacillus Calmette-Guerin (BCG) is a live-attenuated strain of Mycobacterium bovis (M Bovis), the cause of tuberculosis (TB) in cattle, and a member of the Mycobacterium tuberculosis complex (MTBC). Originally developed as a vaccine against tuberculosis by Calmette and Guérin ${ }^{1}$, the use of intravesical BCG in the treatment of bladder cancer was first described in $1976^{2}$. BCG bacillus stimulates the immune system inducing a type IV hypersensitivity reaction in the bladder mucosa, which initiates cell-to-cell cytotoxicity targeting tumour cells. It is the adjunct therapy of choice in the treatment of superficial (non-muscle invasive) bladder cancer, with delay in tumor progression, prevention of tumor recurrence and improvement in survival ${ }^{3,4,5}$. A complete response is achieved in $60 \%$ $95 \%$ of patients, more effective than any chemotherapeutic agent ${ }^{6}$. While usually associated with only mild side effects (such as dysuria, frequency of micturition, malaise and low-grade fever), more lifethreatening systemic infectious complications (such as pneumonitis and hepatitis) can occur ${ }^{7}$. We present a case of disseminated BCGosis which was only discovered at post-mortem, in an elderly man with transitional cell carcinoma. He had risk factors for both MTB and $\mathrm{M}$ bovis infection TB and died in the context of pre-existing cardiac and respiratory dysfunction. Our case highlights: (1) the potential for disseminated BCG-osis to occur after a long latency period and 
(2) the difficulty in rapidly distinguishing $M$ bovis from MTB in the laboratory. This differentiation impacts clinical management, infection control and public health response.

\section{Case Presentation}

An 89-year-old male was admitted to a tertiary referral centre in inner Sydney in December 2015, referred by his gastroenterologist with a 1-month history of low-grade fevers, night sweats, exertional dyspnoea and 8kg weight loss. His past medical history was significant for bladder cancer, an abdominal aortic aneurysm (AAA) repaired in 2012, iron deficiency anaemia, mild chronic obstructive pulmonary disease and past asbestos exposure. He had likely distant past TB exposure from his father and had no evidence for immunesuppression. His only medications were iron supplements and occasional Ventolin inhaler as required.

In September 2006, he developed painless haematuria and was diagnosed with Grade two papillary transitional cell carcinoma (TCC). He underwent transurethral resection of his bladder in October 2006, and then six episodes of weekly instillations of intravesical BCG. In February 2007, biopsy showed Grade three TCC, for which he received a further six weekly instillations. Following subsequent positive biopsies, he was given further intravesical BCG totaling two in January 2009 and six in April/May 2012. Follow-up cystoscopy, performed every six months until March 2015, did not reveal recurrent tumour.

On hospital admission in 2015, he was hypoxaemic, with coarse crackles throughout both lungs and bilateral pitting oedema to his knees. On investigation, he had raised lipase/amylase and C - reactive protein with abnormal liver function tests. His B-type natriuretic peptide was $8130 \mathrm{pg} /$ ml. A high- resolution chest-abdominal-pelvis CT scan showed diffuse ground-glass changes with interlobular septal thickening in keeping with cardiogenic pulmonary oedema. There were also subpleural emphysematous changes in the upper lobes and interstitial fibrosis in the lung bases. The CT also demonstrated multiple foci of calcific lesions throughout both lungs measuring up to $2 \mathrm{~mm}$ in diameter. No axillary, hilar or mediastinal lymphadenopathy was identified. A transthoracic echocardiogram showed mild global cardiomyopathy with an ejection fraction of 45 percent. The patient developed progressive type 1 respiratory failure and despite noninvasive positive pressure ventilation, intravenous loop diuretics for acute decompensated heart failure and broad spectrum antibiotics - Intravenous Ceftriaxone 1 gram once daily OD for the first two days switched to intravenous Ampicillin 1 gram four times daily following a positive urine culture during his admission for Enterococcus faecalis. He died eleven days into his admission despite optimization of his management from the geriatric, cardiology and respiratory teams involved in his care.
At post-mortem, macroscopic examination of the lungs showed bilateral apical emphysematous changes and three possible pleural plaques. The heart showed severe ischaemic heart disease $(>95$ percent occlusion of the right coronary artery and moderate interstitial fibrosis of the left posterior ventricle). The infrarenal metal stent was noted in association with a $6 \mathrm{~cm}$ aortic aneurysm. On microscopic examination, numerous non-necrotising well-formed granulomas, composed of epithelioid histiocytes and intermixed multinucleated giant cells, were found throughout the lungs, spleen and liver. In the lungs, the hilar lymph node section was unremarkable. Two asbestos bodies were identified in the right upper and lower lung lobes (one seen with Perls stain), the left pleural plaque was confirmed on histology and the right lower lobe showed alveoli expanded by suppurative inflammation in keeping with bacterial pneumonia.

On microbiological testing of lung tissue, no acid-fast bacilli were seen on smear but $M$ tuberculosis complex DNA was detected by polymerase chain reaction (PCR), using the Xpert ${ }^{\circledR}$ MTB/RIF (Cepheid) platform, the day after the patient's death. Because of risk factors for both MTB and M bovis exposure, one could not clinically favour either $M$ bovis or MTB as the cause for the positive PCR result. This created concern about potential MTB exposure of hospital patients, ward staff and laboratory staff (antemortem and post-mortem). Post-mortem bacterial lung tissue cultures grew Pseudomonas aeruginosa (possibly pathogenic), Staphylococcus epidermidis and Streptococcus parasanguinis, (both of doubtful clinical significance). In the liver and spleen, no acid-fast bacilli were seen, however, after fifteen days incubation, a mycobacterial species was isolated from both liver and spleen, using the BACTEC ${ }^{\text {тм }}$ MGIT $^{\mathrm{TM}}$ automated mycobacterial detection system (Becton Dickinson). Twenty-eight and thirty days post-mortem, a mycobacterial species was likewise cultured from the left and right lungs respectively. The rapid SD Bioline MPT64 antigen test on the isolate in MGIT broth was positive for MTBC however further speciation required testing at the statewide Mycobacterium Reference Laboratory.

Phenotypic drug susceptibility testing revealed that the mycobacterial species was susceptible to isoniazid, rifampicin and ethambutol but resistant to pyrazinamide, suggesting that the isolate was likely to be $M$. bovis or $M$. bovis (BCG). Genomic deletion analysis is a PCR-based molecular method which looks for the characteristic loss of genetic material by M. bovis and M. bovis (BCG) in specific genetic regions of difference compared with $\mathrm{MTB}^{8}$. Genomic deletion analysis, using a previously published method $^{9}$ was performed on the isolates from our patient's lung, liver, and spleen, and was found to be M. bovis BCG.

\section{Discussion}

This report describes an unusual presentation of 
systemic BCG-osis appearing more than three and a half years after the last BCG instillation and the authors were unaware of the possibility of BCG-osis causing a fatality so long afterwards. A case series of 256 patients treated with intravesical BCG therapy describes a median of 13.5 days before onset of symptoms ${ }^{10}$. Late-presenting BCG disease certainly occurs but usually involves the genitourinary tract and other localised sites, such as osteomyelitis or mycotic aneurysm. The longest interval reported between BCG instillation and presentation with active infection was 17 years in a case of epididymo-orchitis ${ }^{11}$. Spondylodiscitis has been seen 11 years post instillation ${ }^{12}$ and mycotic aneurysms on average discovered two and a half years later ${ }^{13}$. On that note, we have no specific evidence that our patient's aneurysm was infective, but this was not specifically investigated either ante-mortem or postmortem, as the autopsy was completed before the BCG-osis diagnosis was established.

The patient's death was almost certainly multifactorial in aetiology (including severe coronary artery disease, COPD and possible Pseudomonas aeruginosa pneumonia), but it is likely that disseminated BCG-osis was a significant contributing factor. There was proven involvement of the lung, liver and spleen, consistent with blood borne sepsis and dissemination. Contamination of post-mortem cultures and post-mortem translocation of organisms are well recognised phenomena, but Pseudomonas aeruginosa is a cause of hospital acquired pneumonia. Microbiological blood cultures showed no staphylococcal, streptococcal or Pseudomonal bacteraemia. Furthermore, it was stated by Reidel that post-mortem bacterial transmigration may have little influence on post-mortem microbiologic cultures if the autopsy is performed within the first 24 to $48 \mathrm{~h}$ after death, which is the circumstance in our case ${ }^{14}$. Cardiac death is known to be increased in patients with pulmonary infections. A similar case was reported by Madentzogolou et al., in which a man with disseminated BCG infection died with evidence at autopsy of severe coronary artery disease and myocardial infarction ${ }^{15}$.

It is unknown in these circumstances if earlier diagnosis and treatment of his BCG-osis would have changed his ultimate clinical outcome. The treatment for disseminated BCG-osis is not well established however it is recommended to consist of aggressive anti-tuberculous treatment considering the susceptibility pattern ${ }^{16}$. Corticosteroids have also been used to treat BCG sepsis in the past ${ }^{17,18}$. Even with treatment for BCG-osis, it is unclear whether this patient would have survived the acute phase.

In the 3-4-week period between positive MTBC PCR results and final speciation, there were significant concerns regarding hospital infection control and contact tracing as the risks to exposed contacts differs greatly between MTB (with human-to-human, the usual mode of transmission) and $M$. bovis (BCG) unlikely to cause human-to-human transmission. As there were no pregnant women, infants or immunosuppressed patients among those potentially exposed, formal contact tracing was deferred until speciation was available.

Neither MTB nor BCG-osis were suspected ante-mortem in our case Therefore, no airborne precautions were instituted, and he received non-invasive positive pressure ventilatory support in an open bay, high dependency area, potentially exposing a number of other patients. Likewise, autopsies are known be high risk procedures for acquisition of infection ${ }^{19}$, making negative pressure rooms and appropriate personal protective equipment necessary for those conducting post-mortem examinations.

There are some reported cases of person-to-person transmission of $M$. bovis despite its usual mode of transmission being either cattle to human, animal to human or most commonly unpasteurised dairy products ${ }^{20}$, 21. Nosocomial transmission of BCG-osis has been described. Three patients being treated for haematological malignancies were described as likely to have had their cancer chemotherapy contaminated with BCG vaccine at an institution in the United States of America ${ }^{22}$. In addition, in a subsequent study $M$. bovis BCG person to person spread was suspected from these patients to $5 \%(2 / 41)$ of healthcare workers examined ${ }^{23}$.

Severe adverse events due to local instillation of Bacillus Calmette-Guérin (BCG) are uncommon. In up to $90 \%$ of patients with BCG-osis symptoms are usually very mild $^{24}$. Perez-Jacoiste Asin et al. identified three factors linked with increased mortality in patients with BCG infection: age $>65$ years old, disseminated infection and vascular involvement. It is likely our patient had all three risk factors ${ }^{10}$. Traumatic catheterisation, diabetes and concurrent cystitis were found to be predictors for those patients at risk of developing BCG infection ${ }^{8}$. Our patient had no documented evidence of these issues.

Guidelines recommend pre-treatment assessment of the risks of intravesical BCG instillation to be carried out by clinicians before embarking on this therapy, especially in patients with known immune-suppression ${ }^{25}$.

Without the appropriate performance of a post-mortem in this case the diagnosis of BCG-osis would not have been elucidated. The use of autopsy as a clinical tool should not be overlooked as we move forward into the molecular diagnostic era.

\section{Take Home Messages}

- BCG instillation of the bladder for transitional cell carcinoma should be acknowledged as posing a risk of systemic spread

- All physicians who instill BCG into the bladder should 
be aware of the complications and contraindications to the procedure

- Disseminated BCG-osis can occur many years following bladder instillation

\section{Acknowledgements}

We the authors, would like to thank the Tuberculosis and Microbiology services at St Vincent's Hospital, Sydney in addition to the Institute of Clinical Pathology and Medical Research (ICPMR) at Westmead Hospital.

\section{References}

1. Calmette A. Preventive Vaccination Against Tuberculosis with BCG. Proc R Soc Med. 1931 Sep; 24(11): 1481-90.

2. Schellhammer PF, Ladaga LE, Fillion MB. Bacillus Calmette-Guerin for superficial transitional cell carcinoma of the bladder. Journal of Urology. 1986; 135: 261-4.

3. Guallar-Garrido S. Bacillus Calmette-Guerin immunotherapy for bladder cancer overview of BCG immunotherapy: An Update Immunotargets Ther. 2020; 9: 1-11.

4. Prescott S, Jackson AM, Hawkyard SJ, et al. Mechanisms of action of intravesical bacille Calmette-Guerin: local immune mechanisms. Clin Infect Dis. 2000; 31 (suppl 3): S91-3.

5. Nseyo UO, Lamm DL. Immunotherapy of bladder cancer. Semin Surg Oncol. 1997; 13: 342-349.

6. Alcaraz A. Bladder Cancer: Highlights from 2006 European Association of Urology European Urology Supplements. 2007; 6: 737- 744.

7. Lamm DL. Incidence and treatment of complications of bacillus Calmette-Guerin immunotherapy. Urology Clinical North America. 1992; 19: 565-72.

8. Parsons LM, Brosch R, Cole ST, et al. Rapid and Simple Approach for Identification of Mycobacterium tuberculosis Complex Isolates by PCRBased Genomic Deletion Analysis J Clin Microbiol. 2002; 40(7): 2339-2345.

9. Huard RC, Lazzarini LCDO, Butler WR, et al. PCR-based method to differentiate the subspecies of the Mycobacterium tuberculosis complex on the basis of genomic deletions. J Clin Microbiol. $2003 \mathrm{Apr}$; 41(4): 1637-50.

10. Perez-Jacoiste Asín MA, Fernandez-Ruiz M, Lopez-Medrano F, et al Bacillus Calmette-Guerin (BCG) Infection Following Intravesical BCG Administration as Adjunctive Therapy for Bladder Cancer: Incidence, risk factors \& outcome in a single institution \& review of the literature. Medicine Baltimore. 2014 Oct; 93(17): 236-54.

11. Salvador R, Vilana R, Bargallo X, et al. Tuberculous Epididymo-orchitis after Intravesical BCG Therapy for Superficial Bladder carcinoma. Journal of Ultrasound Medicine. 2007; 26: 671-674.
12. Mavrogenis AF, Sakellariou VI, Tsiodras S, et al. Late Mycobacterium bovis spondylitis after intravesical BCG therapy. Joint Bone Spine. 2009; 76: 296-300.

13. Coddington ND, Sandberg JK, Yang C, et al. Mycotic Aneurysm after Bacillus Calmette-Guérin Treatment: Case Report and Review of the Literature. Case Reports in Urology Volume. 2017; 4508583: 4.

14. Riedel S. The Value of Postmortem Microbiology Cultures J Clin Microbiol. 2014 Apr; 52(4): 1028- 1033.

15. Madentzogolo MS. Lethal BCG-osis, in the context of superficial urothelial bladder carcinoma, diagnosed in autopsy, Egyptian Journal of Forensic Sciences. 2015.

16. Al Arishi HM. Clinical features and outcome of eleven patients with disseminated bacille Calmette-Guerin (BCG) infection Ann Saudi Med. 1996; 16 (5): 512-516.

17. Steg A, Leleu C, Debre B, et al. Systemic bacillus Calmette-Guerin infection in patients treated by intravesical BCG therapy for superficial bladder cancer. Prog Clin Biol Res. 1989; 310: 325.

18. Wittes R, Klotz L, Kosecka U. Severe bacillus Calmette-Guerin cystitis responds to systemic steroids when antituberculous drugs and local steroids fail. J Urol. 1999; 161(5): 1568.

19. Templeton GL, Illing LA, Young L, et al. Transmission of Mycobacterium tuberculosis at the Bedside and during Autopsy. Ann Intern Med. 1995; 122(12): 922-925.

20. Rua-Domenech R. Human Mycobacterium bovis infection in the United Kingdom: Incidence, risks, control measures and review of the zoonotic aspects of bovine tuberculosis. Tuberculosis (Edinb). 2006 Mar; 86(2): 77-109. Epub 2005 Oct 28.

21. Waecker NJ Jr, Stefanova R, Cave MD, et al. Nosocomial Transmission of Mycobacterium bovis Bacille Calmette-Guerin to Children Receiving Cancer Therapy and to Their Health Care Providers. Clinical Infectious Diseases. February 2000; 30(2): 356-362. https://doi. org $/ 10.1086 / 313652$

22. Evans JT, Smith EG, Banerjee A, et al. 2007 Cluster of human tuberculosis caused by mycobacterium bovis: evidence for person-toperson transmission in the UK. Lancet. 2007; 369: 1270-76.

23. Stone MM, Vannier AM, Storch SK, et al. Brief report: menigitis due to iatrogenic BCG infection in two immunocompromised children. N Engl J Med. 1995; 333: 561-563.

24. Waecker NJ, Stefanova R, Cave MD, et al. Nosocomial Transmission of Mycobacterium bovis Bacille Calmette-Guerin to Children Receiving Cancer Therapy and to Their Health Care Providers. Clinical Infectious Diseases. 2000; 30: 356-362.

25. Babjuk M, Böhle A, Burger M, et al. European Association of Urology guidelines on non-muscle-invasive urothelial carcinoma of the bladder update 2016. Eur Urol. 2016; 64: 639-53. 